\title{
THE EFFECT OF MULTIPLE-DOSE PAROXETINE ON THE PHARMACOKINETIC PROFILE OF CARVEDILOL IN RATS
}

\author{
MARIA B. ABRUDAN ${ }^{1}$, DANA M. MUNTEAN ${ }^{1 *}$, MARIA A. NEAG $^{2}$, LAURIAN VLASE $^{1}$ \\ and ANA-MARIA GHELDIU'
}

\begin{abstract}
${ }^{1}$ University of Medicine and Pharmacy "Iuliu Haţieganu”, Faculty of Pharmacy, Department of Pharmaceutical Technology and Biopharmaceutics, Victor Babes 41, RO-400012, Cluj-Napoca, Romania

${ }^{2}$ University of Medicine and Pharmacy "Iuliu Haţieganu", Faculty of Medicine, Department of Pharmacology, Toxicology and Clinical Pharmacology, Marinescu 23, RO-400337, Cluj-Napoca, Romania

Abstract: The aim of the present study was to evaluate the effect of multiple-dose paroxetine upon the pharmacokinetic profile of carvedilol in rats. Carvedilol was orally administrated in rats $(3.57 \mathrm{mg} / \mathrm{kg}$ body mass (b.m.)) in the absence of paroxetine or after a pre-treatment with multiple oral doses of paroxetine $(8.6 \mathrm{mg} / \mathrm{kg}$ b.m.). The plasma concentrations of carvedilol were estimated by high-performance liquid chromatographytandem mass spectrometry. After carvedilol co-administration with paroxetine, an approximately 4.5-fold increase in the exposure of carvedilol was observed, considering the significantly elevated value of $\mathrm{AUC}_{0-\infty}$. Furthermore, an increase of $72 \%$ of peak plasma concentration was found, as well as an augmentation by $91 \%$ of the half life time of carvedilol was observed. Paroxetine co-administration led to a significant alteration of carvedilol's pharmacokinetic profile in rats, these effects could be explained by the existence of a drug-drug interaction mediated by CYP2D6 inhibition.
\end{abstract}

Keywords: carvedilol, paroxetine, pharmacokinetics, cytochrome P450s, preclinical study

As a third generation beta-blocker, carvedilol is currently approved for the treatment of hypertension, congestive heart failure, ischemic heart disease and angina $(1,2)$. The chemical structure is that of an arylethanolamine, being clinically used as a racemic mixture of S-(-) and R-(+)-enantiomer, each of the enantiomers possessing distinct pharmacodynamic profile (3). The S-(-)-enantiomer displays both $\beta 1$ - and $\alpha 1$-adrenoreceptor-blocking activity, whereas the $\mathrm{R}-(+)$-enantiomer possesses alpha-antagonist activity at equal potency to S-(-)-enantiomer (4). Carvedilol presents cardioprotective, vasodilatative and anti-proliferative activity due to its nitric oxide release effect (5). In addition, the active metabolite 4'-hydroxyphenyl carvedilol is 13 times more potent than the parent compound and therefore is supposed to contribute to a certain extent to the clinical effects of carvedilol (6). This active metabolite and 5'-hydroxyphenyl carvedilol are formed by oxidative biotransformation mediated by isoenzyme CYP2D6 (6). To a lesser extent, CYP2C9 and CYP1A2 mediate carvedilol metabolism to other metabolites (7). After oral administration, carvedilol displays rapid and extensive absorption with an absolute bioavailability, not affected by concomitant food intake, ranging from $25 \%$ to $35 \%$ due to intensive first-pass metabolism $(1,2)$.

Paroxetine, of all the selective serotonin reuptake inhibitors (SSRIs), possesses the highest specificity for serotonin receptors (8). It is indicated for the treatment of major depressive disorder and a wide range of anxiety disorders (9). It displays good absorption following oral administration, with the steady-state plasma concentration attained after 7 to 14 days of multiple dose regimen (10). It is highly bound to plasma proteins (95\%) and is subjected to extensive metabolism in the liver, mainly by methylation and oxidation to metabolites which were found to exhibit very weak in vitro inhibitory potency on serotonin reuptake mechanisms (11). The main isoenzyme involved in the biotransformation of paroxetine is CYP2D6 (8). Moreover, paroxetine is known as a potent inhibitor of CYP2D6, thus it displays nonlinear pharmacokinetics (12) and it can also alter the pharmacokinetic profile of co-administered drugs which are also substrates for CYP2D6, such as carvedilol.

* Corresponding author: e-mail: dana.muntean@umfcluj.ro 
Considering that depressive disorders are acknowledged to be highly prevalent among cardiovascular patients (13) and depression is a risk factor for cardiovascular diseases (14), the association of an antidepressant and beta-blocker is very likely to be often encountered in pluri-drug therapeutic schemes for patients suffering from the aforementioned diseases. Hence, the aim of this study was to investigate the association carvedilol-paroxetine in rats from the pharmacokinetic point of view and, if the metabolism-based interaction is present, to assess its magnitude and evaluate its impact for clinical practice.

\section{EXPERIMENTAL}

\section{Chemicals and reagents}

Carvedilol and paroxetine were bought from Moehs (Rubí, Spain). HPLC-grade acetonitrile, 98\% formic acid and methanol of analytical-reagent grade were purchased from Merck KGaA (Darmstadt, Germany). Heparin sodique $25000 \mathrm{UI} / 5 \mathrm{~mL}$ (5000 UI/mL) was bought from Panpharma Laboratoires (France).

The devices used in this study were: BASi Culex ABC® - Automatic Blood Collector (BASi, Indiana, USA), an Agilent 1100 series - HPLC system (with binary pump, autosampler, thermostat) (Agilent Technologies, USA), coupled with a Bruker Ion Trap VL (Bruker Daltonics GmbH, Germany).

\section{Animal treatment}

In this preclinical study, a number of 12 white male Wistar rats were included. Their weights were between 300 and 370 grams and were purchased from the Experimental Animal Centre (ClujNapoca, Romania). The Ethics Committee of the University of Medicine and Pharmacy "Iuliu Hatieganu", Cluj-Napoca, Romania, reviewed and approved the working protocol prior to the beginning of the preclinical trial.

An acclimatization period of seven days was employed for the rats before the study, by placing each of them in solitary cages and allowing free access to tap water ad libitum and specific rat diet. Moreover, they were kept in optimal conditions: clean room under constant ambient temperature (22 $\pm 2^{\circ} \mathrm{C}$ ) and humidity, with $12 \mathrm{~h}$ light - dark cycles. The study was conducted in accordance with the specific regulations and amendments as it follows: the 43/2014 Law stating the protection of animals used for scientific research purpose published in "Monitorul Oficial" (Romania) and the "Guiding
Principles in the Use of Animals in Toxicology" adopted by the Society of Toxicology (USA).

Before being connected to BASi Culex $\mathrm{ABC}^{\circledR}$, each rat was cannulated on his left femoral vein with the purpose of precise blood sampling at different time intervals after drug administration. The surgery was done under anesthesia, with a mixture of ketamine, xylazine and diazepam $(1: 1: 1)$. The surgery was performed $24 \mathrm{~h}$ before first drug administration (period 1), in order to have the anesthetic mixture eliminated.

\section{Study design}

The design of the study was that of an openlabel, sequential preclinical trial, which consisted of two periods. During the first study period (Reference), each rat received an oral dose of 3.57 $\mathrm{mg} / \mathrm{kg}$ body mass (b.m.) carvedilol, whilst during the second period of study (Test) the same dose of carvedilol (3.57 mg/kg b.m.) was orally co-administered with $8.6 \mathrm{mg} / \mathrm{kg}$ b.m. paroxetine. Between the periods of study, the rats were treated for 5 consecutive days with an oral dose of $8.6 \mathrm{mg} / \mathrm{kg}$ b.m. paroxetine, given twice daily, in order to reach the steady-state plasma concentration of this inhibitor.

The solutions for oral administration of carvedilol $(1 \mathrm{mg} / \mathrm{mL})$ and paroxetine $(4 \mathrm{mg} / \mathrm{mL})$ were prepared with reference standards of pharmaceutical purity, dissolved in a saline solution; afterwards, a sterilizing membrane was employed for filtration of the solutions.

During each study period, a series of $200 \mu \mathrm{L}$ venous blood samples were taken. They were drawn into heparinized tubes after carvedilol administration, at specified time intervals: 5, 10, 15, 20, 30, 45 min and 1, 2, 4, 8, 12, 18, 24, $30 \mathrm{~h}$ after carvedilol administration.

\section{Sample preparation}

The sample preparation was done in Eppendorf tubes. $0.3 \mathrm{~mL}$ methanol was added over $0.1 \mathrm{~mL}$ blood. Afterward, the tubes were vortex-mixed for $10 \mathrm{~s}$ and centrifuged for $5 \mathrm{~min}$ at $10000 \mathrm{RPM}, 9167 \mathrm{~g}$. The supernatant was subsequently placed in an autosampler vial, the injection volume in the chromatographic system consisting of $15 \mu \mathrm{L}$.

\section{High-performance liquid chromatography (HPLC) assay}

The plasma concentrations of carvedilol were determined by a validated liquid chromatography-tandem mass spectrometry method. The analytical method for carvedilol determination was not enantioselective. The HPLC system was an Agilent 1100 
series (binary pump, autosampler, thermostat) (Agilent Technologies, USA) and was coupled with a Bruker Ion Trap VL (Bruker Daltonics GmbH, Germany). It was used a Zorbax SB-C18 chromatographic column (50 mm $\times 2.1 \mathrm{~mm}$ i.d., $3.5 \mu \mathrm{m})$ (Agilent Technologies). The mobile phase consisted of a $34: 66(\mathrm{~V} / \mathrm{V})$ mixture of acetonitrile and $0.2 \%(\mathrm{~V} / \mathrm{V})$ formic acid in water. The flow rate was of $0.5 \mathrm{~mL} / \mathrm{min}$, and the thermostat temperature was set at $42^{\circ} \mathrm{C}$. The mass spectrometry detection was in multiple reaction monitoring mode, positive ions, using an electrospray ionization source. The ion transitions monitored were $\mathrm{m} / \mathrm{z}$ 222, 224, 283 from $\mathrm{m} / \mathrm{z} 407$ for carvedilol (multiple reaction monitoring mode) using an atmospheric pressure electrospray ionization ion source (polarity: positive, nebulizer 40 psi (nitrogen), dry gas nitrogen at $9 \mathrm{~L} / \mathrm{min}$, dry gas temperature $350^{\circ} \mathrm{C}$, ion charge control target: 50000, charge control: ON. $(15,16)$.

The calibration curve of carvedilol was linear at a concentration range of $6-577 \mathrm{ng} / \mathrm{mL}$ plasma, with a correlation coefficient greater than 0.997 . The method shows a good linearity $(r>0.993)$, intra- and inter-day precision $(\mathrm{CV} \%<11.3 \%)$ and accuracy (bias < $12.7 \%$ ) over the range of calibration curve. The lower limit of quantification (LLOQ) was $6 \mathrm{ng} / \mathrm{mL}$ and the overall recovery was between $92.3-106.4 \%$.

\section{Pharmacokinetic analysis}

For the determination of the pharmacokinetic (PK) parameters of carvedilol, given alone or in combination with paroxetine, the non-compartmental pharmacokinetic analysis method was employed. The calculation was carried out with Phoenix WinNonlin 6.3 software (Pharsight Co., Mountain View, CA, USA). The peak plasma concentration $\left(\mathrm{C}_{\max }, \mathrm{ng} / \mathrm{mL}\right)$ and the corresponding time to reach the maximum concentration $\left(\mathrm{t}_{\max }, \mathrm{h}\right)$ were estimated by visual inspection of individual plasma concentration versus time profiles. The area under the concentration-time curve from time zero to the last quantifiable concentration $\left(\mathrm{AUC}_{0-\mathrm{t}}\right)$ was calculated with the linear trapezoidal rule. The area extrapolated to infinity $\left(\mathrm{AUC}_{0-\infty}\right)$ was obtained by adding $\mathrm{C}_{\mathrm{t}} / \mathrm{k}_{\mathrm{el}}$ to $\mathrm{AUC}_{0-\mathrm{t}}$, where $\mathrm{C}_{\mathrm{t}}$ is the last measurable plasma drug concentration, while $\mathrm{k}_{\mathrm{el}}$ is the first order elimination rate constant. The $\mathrm{k}_{\mathrm{el}}$ was determined from semi-logarithmic plot corresponding to first order elimination kinetics, by the least-square regression of plasma concentration versus time data points lying in the terminal region. The half-life time of carvedilol was determined as $0.693 / \mathrm{k}_{\mathrm{el}}$.

A compartmental analysis using the predefined one or two compartment models was performed

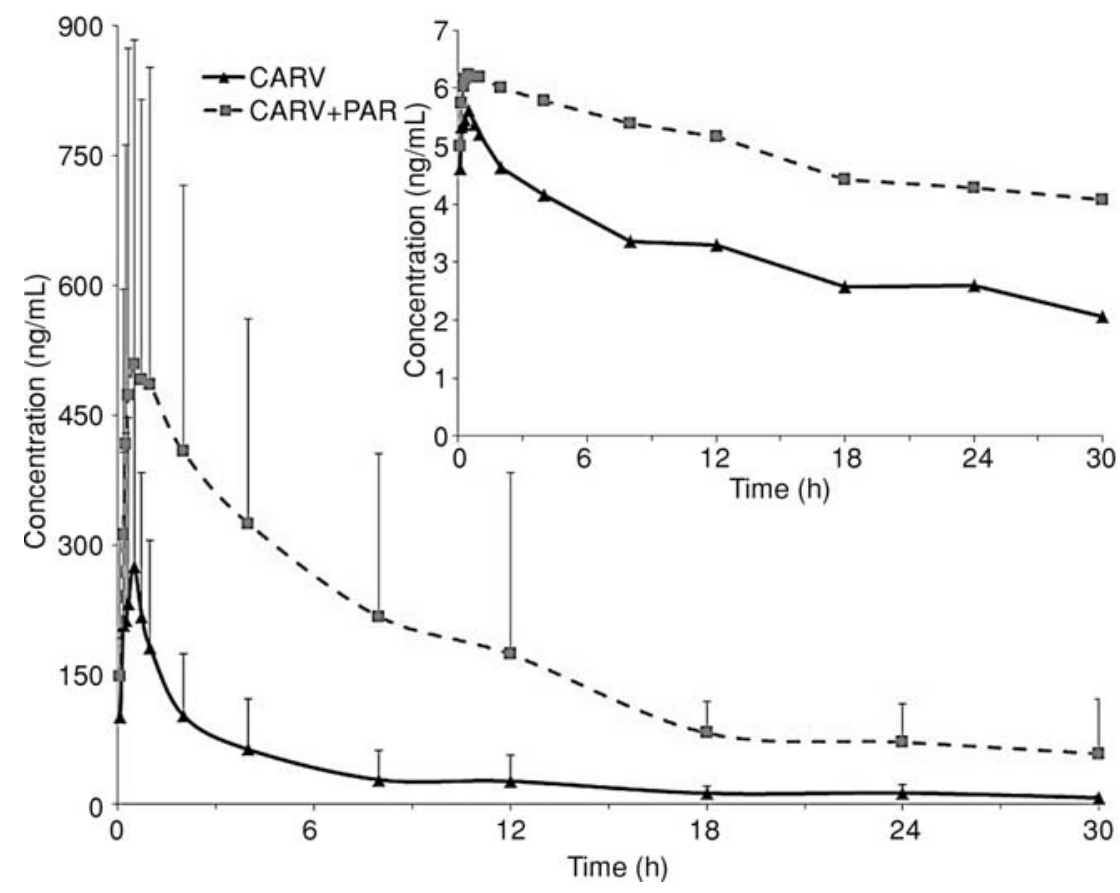

Figure 1. Mean \pm SD plasma concentrations of carvedilol, after oral administration of single dose carvedilol (3.57 mg/kg b.m.) alone ( $\mathbf{\Delta})$ or in combination with paroxetine $(\mathbf{\square})$, after pre-treatment with paroxetine for 5 days $(\mathrm{n}=12)$ 
using Phoenix WinNonlin 6.3 software (Pharsight Co., Mountain View, CA, USA). The Akaike information criteria (AIC) was used to compare these models and the distribution model was chosen based on the lower AIC value obtained.

\section{Statistical analysis}

For the statistical analysis, the Phoenix WinNonlin software version 6.3 was employed (Pharsight Co., Mountain View, CA, USA). The data are further presented as the mean value \pm standard deviation (S.D.). The pharmacokinetic parameters from the Reference (1) and Test period (2) were compared using a one-way analysis of variance test (ANOVA), a $p$ value below 0.05 was considered for statistical significance. For $\mathrm{t}_{\max }$, the statistical significance between study periods was assessed by the nonparametric Friedman test.

\section{RESULTS}

The mean plasma concentration-time profiles for carvedilol, following oral administration, of carvedilol alone or in combination with paroxetine, after a 5 days pretreatment with the enzymatic inhibitor, are depicted in Figure 1.

As illustrated in Figure 1 and Table 1, paroxetine had a major impact upon the pharmacokinetic profile of carvedilol in rats. More precisely, the mean peak plasma concentration $\left(\mathrm{C}_{\max }\right)$ of carvedilol $(323.19 \pm 232.55$ vs. $554.64 \pm 369.55 \mathrm{ng} / \mathrm{mL})$ was approximately 1.7 -fold higher after pretreatment with paroxetine than the levels registered after carvedilol was administered alone.

Paroxetine therapy was associated with a 4.4fold increase in the $\mathrm{AUC}_{0-\infty}$ values of carvedilol $(1163.97 \pm 695.79$ vs. $5223.57 \pm 3430.92 \mathrm{ng} \times$ $\mathrm{hr} / \mathrm{mL}$ ). Apart from the previously mentioned PK parameters for which the differences registered for each treatment period were statistically significant according to ANOVA test, the following PK parameters displayed statistically significant values between study periods: the elimination rate constant $\left(\mathrm{k}_{\mathrm{el}}\right)$, the half-life time $\left(\mathrm{t}_{1 / 2}\right)$, the apparent clearance $\left(\mathrm{Cl} \_\mathrm{F}\right)$ and the apparent volume of distribution (Vz_F). The time to reach the peak plasma concentration did not differ in a statistically significant manner, according to the Friedman test.

Regarding the compartmental PK analysis, for the mono-compartment model, a mean AIC value of 78.28 was obtained, while for the two-compartment model the mean value of AIC was 62.74. Using AIC value to discriminate between models, we can assume that drug distribution follows the characteristics of two-compartment model. For this study, the carvedilol's pharmacokinetics in rats can be better described and predicted by a two-compartment model, when after a period of time, a distribution equilibrium is achieved between the central and peripheral compartments.

In the present study, the non-compartmental PK analysis along with the statistical evaluation by means of ANOVA and Friedman test confirmed that a metabolism-based drug-drug interaction was present between carvedilol and paroxetine and the increased exposure to carvedilol (substrate of CYP2D6) was attributed to paroxetine pretreatment and concomitant administration during Test period, the latter being the CYP2D6 inhibitor.

\section{DISCUSSION}

The information regarding the drug-drug interactions of carvedilol are rather scarce, therefore the present study will bring a valuable update for the safety profile of this beta-blocker. When other antidepressants were co-administered with carvedilol in rats, the interactions resulted in markedly elevated

Table 1. Main pharmacokinetic (PK) parameters of carvedilol in rats $(\mathrm{n}=12)$ after single oral dose of $3.57 \mathrm{mg} / \mathrm{kg}$ b.m. of carvedilol, before and after treatment with paroxetine $(8.6 \mathrm{mg} / \mathrm{kg} \mathrm{b.m.)} \mathrm{for} 5$ days and the results of statistical test used for comparison

\begin{tabular}{|l|c|c|c|}
\hline $\begin{array}{c}\text { PK parameter } \\
(\text { mean } \pm \mathrm{SD})\end{array}$ & Carvedilol & Carvedilol + Paroxetine & $p^{*}$ value (ANOVA) \\
\hline $\mathrm{C}_{\max }(\mathrm{ng} / \mathrm{mL})$ & $323.19 \pm 232.55$ & $554.64 \pm 369.55$ & 0.04611 \\
\hline $\mathrm{t}_{\max }(\mathrm{h})$ & $2.3 \pm 3.92$ & $0.92 \pm 1.14$ & Friedman, NS* \\
\hline $\mathrm{AUC}_{0-\infty}(\mathrm{ng} * \mathrm{hr} / \mathrm{mL})$ & $1163.97 \pm 695.79$ & $5223.57 \pm 3430.92$ & 0.00001 \\
\hline $\mathrm{k}_{\mathrm{el}}(1 / \mathrm{h})$ & $0.18 \pm 0.15$ & $0.07 \pm 0.03$ & 0.00411 \\
\hline $\mathrm{t}_{1 / 2}(\mathrm{~h})$ & $5.67 \pm 3.07$ & $10.85 \pm 4.25$ & 0.00411 \\
\hline $\mathrm{Cl} \_\mathrm{F}(\mathrm{mL} / \mathrm{h} / \mathrm{kg})$ & $3.96 \pm 2.13$ & $0.78 \pm 0.41$ & $<0.00001$ \\
\hline $\mathrm{Vz} \_\mathrm{F}(\mathrm{mL} / \mathrm{kg})$ & $30.02 \pm 21.56$ & $11.64 \pm 6.65$ & 0.00619 \\
\hline
\end{tabular}

*NS- statistically non-significant 
plasma concentrations of this beta-blocker. For instance, bupropion produced an increase of $40 \%$ for the $\mathrm{C}_{\max }$ of carvedilol (16) while sertraline co-administration led to an increase of the same PK parameter by $150 \%$ and citalopram pretreatment and co-administration resulted in 2.3-fold higher $\mathrm{C}_{\max }$ for carvedilol (17). Moreover, citalopram and bupropion produced an increase of 2.7-fold for area under the curve of carvedilol $(16,17)$ while sertraline co-administration led to a 7.7-fold increase of this PK parameter (15).

Apart from the previously mentioned interactions with different antidepressants, other interactions were studied for carvedilol. For instance, during co-administration with digitoxin, phenprocoumon, glyburide, hydrochlorothiazide, and torsemide no change was registered in the pharmacokinetic profile of carvedilol $(18,19)$. On the other hand, the concomitant use of rifampicin $(600 \mathrm{mg}$ for 12 days) led to a $70 \%$ decrease in area under the curve and $\mathrm{C}_{\max }$ of carvedilol (20), while cimetidine co-administration (1000 mg/day) produced a $30 \%$ increase in area under the curve but did not modify the $\mathrm{C}_{\max }$ of carvedilol (21).

Taking into consideration that the isoenzyme CYP2D6 is mainly involved in carvedilol's biotransformation and that after pretreatment and concomitent administration of paroxetine the main PK parameters of carvedilol were significantly modified, the mechanism of interaction for this drug association is the inhibition of CYP2D6 by paroxetine (7, 12). The PK interaction was assessed in an appropriate animal model (white Wistar rats), considering that the main purpose of this study was to investigate whether these drugs interact (carvedilol and paroxetine) and what is the magnitude of their interaction. The extrapolation of this outcome to humans could be difficult taking into account the inter-species differences and therefore additional studies on human subjects are advisable (22). Nevertheless, until further investigation caution should be recommended when this drug association is needed in clinical practice for patients affected by both cardiovascular diseases and depressive symptoms.

\section{CONCLUSIONS}

The purpose of this study was to provide new data regarding the safety profile of carvedilol by investigating its association with paroxetine. The pharmacokinetic profile of carvedilol after a single oral dose of paroxetine was statistically significant altered by the multiple-dose pretreatment with paroxetine according to ANOVA results, a value of $p$ being below 0.05 for all the evaluated PK param- eters of carvedilol, expect $t_{\max }$. The increased exposure to carvedilol is observed on the basis of CYP2D6 inhibition mediated by paroxetine and could have implications for clinical practice.

Under the restriction that the steady-state concentrations were obtained only for paroxetine and that the study was performed in rats as the most appropriate animal model, a relevant and definite conclusion for human patients could not be proven. However, considering the magnitude of the PK interaction, caution should be considered until further studies are performed in order to confirm the results of this study in humans.

\section{Acknowledgement}

This work was supported by CNCS Romania project PNII-RU-TE-2014-4-0242.

\section{Disclosure statement}

The authors declare that there are no conflicts of interest.

\section{REFERENCES}

1. Morgan T.: Clin. Pharmacokinet. 26, 335 (1994).

2. Dulin B., Abraham W.T.: Am. J. Cardiol. 93, 3B (2004).

3. Phoung N.T., Lee B.J., Choi J.K., Kang J.S., Kwon K.I.: Arch. Pharm. Res. 27, 973 (2004).

4. Nichols A.J., Sulpizio A.C., Ashton D.J., Hieble J.P., Ruffolo Jr R.R.: Chirality 1, 265 (1989).

5. Vanhoutte P.M., Gao Y.: Curr. Opin. Pharmacol. 13, 265 (2013).

6. Wang Z., Wang L., Xu R.A., Zhan Y.Y., Huang C.K. et al.: Drug. Des. Devel. Ther. 8, 1909 (2016).

7. Oldham H.G., Clarke S.E.: Drug. Metab. Dispos. 25, 970 (1997).

8. Wagstaff A.J., Cheer S.M., Matheson A.J., Ormrod D., Goa K.L.: Drugs 62, 655 (2002).

9. Wagstaff A.J., Cheer S.M., Matheson A.J., Ormond D., Goa K.L.: CNS Drugs 16, 425 (2002).

10. Gunasekara N.S., Noble S., Benfield P.: Drugs 55, 85 (1998).

11. Caccia S.: Clin. Pharmacokinet. 34, 281 (1998).

12. Mikami A., Ohtani H., Hori S., Sawada Y.: Int. J. Clin. Pharmacol. Ther. 51, 374 (2013).

13. Dimos A.K., Stougiannos P.N., Kakkavas A.T., Trikas A.G.: Hellenic J. Cardiol. 50, 410 (2009). 
14. Baune B.T., Stuart M., Gilmour A. et al.: Gen. Hosp. Psychiatry 34, 478 (2012).

15. Abrudan M.B., Muntean D.M., Neag M.A., Vlase L., Gheldiu A.M.: Farmacia 65, 557 (2017).

16. Abrudan M.B., Muntean D.M., Gheldiu A.M., Neag M.A., Vlase L.: Pharmacology 99, 139 (2017).

17. Abrudan M.B., Muntean D.M., Popa D.S., Gheldiu A.M., Neag M.A., Vlase L.: Pharmacology 100, 301 (2017).

18. Harder S., Brei R., Caspary S., Merz P.G.: Eur. J. Clin. Pharmacol. 44, 583 (1993).
19. Van der Does R., Widmann L., Uberbacher H.J., Horrmann M., Machwirth M., Stienen U.: Eur. J. Clin. Pharmacol. 38, S147 (1990).

20. Giessmann T., Modess C., Hecker U., Zschiesche M., Dazert P. et al.: Clin. Pharmacol. Ther. 75, 213 (2004).

21. Keating G.M., Jarvis B.: Drugs 63, 1697 (2003).

22. Martignoni M., Groothuis G.M.M., de Kante R.: Expert. Opin. Drug. Metab. Toxicol. 2, 875 (2006).

Received: 25. 10. 2017 